\title{
A SEARCH FOR OPTICAL-UV FADING OF CENTRAL STARS
}

\author{
B. ALTNER \\ Applied Research Corporation (ARC), Landover, MD USA \\ and \\ S.R. HEAP \\ Laboratory for Astronomy and Solar Physics \\ NASA Goddard Space Flight Center, Greenbelt, MD USA
}

\begin{abstract}
We derive estimates of the masses of planetary nebulae central stars (CSPN), based on their rate of optical-UV fading.

As a central star evolves to higher temperatures, a decreasing fraction of its light is emitted in the optical-UV region of the spectrum. The rate of fading is extremely sensitive to the stellar mass. After reprocessing early IUE spectra and correcting later spectra for the known rate of camera sensitivity degradation (Garhart 1992, IUE Newsletter, 47), we are able to detect evidence of fading in a number of CSPN at a level greater than the minimum detection threshold $(\sim 4 \%)$. The first five columns in the following Table list the star and its type, photometric variability characteristics (Bond 1992, priv. comm.), temperature (in $\mathrm{kK}$ ), and number of IUE spectra used. The sixth column lists the the mean rate of fading in selected wavelength bands free of nebular emission and camera defects. The last two columns show the corresponding stellar masses, calculated by interpolating within the hydrogen-burning sequences of Wood and Faulkner (1986, Ap. J., 307, 659: W \& F) and Blöcker and Schönberner (1990, Astron. Astrophys., 240, L11: B \& S), respectively.
\end{abstract}

\begin{tabular}{lccrcccc}
\hline \hline Central Star & Type & Var? & $\mathrm{T}_{3}$ & $N_{\mathrm{SWP}}$ & $\begin{array}{c}\text { Fading Rate } \\
\text { (\%/decade) }\end{array}$ & $\begin{array}{c}\text { Mass }\left(M_{\odot}\right) \\
\text { W \& F }\end{array}$ & $\begin{array}{c}\text { Mass }\left(M_{\odot}\right) \\
\text { B \& S }\end{array}$ \\
\hline BD +30 3639 & WC9 & - & $26:$ & 4 & $+3.3 \pm 1.6$ & - & - \\
He 2-131 & O7f-eq & - & 27 & 2 & $-4.1 \pm 2.4$ & $0.74_{-0.05}^{+0.05}$ & $0.71_{-0.05}^{+0.03}$ \\
He 2-138 & BC OIa & - & 27 & 3 & $-8.1 \pm 1.2$ & $0.78_{-0.04}^{+0.04}$ & $0.74_{-0.02}^{+0.02}$ \\
NGC 40 & WC8 & Irreg. & $31:$ & 3 & $-4.1 \pm 1.5$ & $0.73_{-0.03}^{+0.02}$ & $0.70_{-0.03}^{+0.02}$ \\
IC 418 & O6f & $\prime \prime$ & 36 & 5 & $-8.9 \pm 3.3$ & $0.77_{-0.03}^{+0.03}$ & $0.73_{-0.03}^{+0.02}$ \\
IC 4593 & O7f & Irreg. & 40 & 4 & $-8.5 \pm 1.1$ & $0.76_{-0.01}^{+0.01}$ & $0.72_{-0.01}^{+0.01}$ \\
NGC 2392 & O6f & Const. & 47 & 6 & $-5.5 \pm 1.5$ & $0.74_{-0.02}^{+0.02}$ & $0.70_{-0.02}^{+0.01}$ \\
NGC 6891 & O3f & Irreg. & 50 & 5 & $-1.3 \pm 1.3$ & - & - \\
NGC 6826 & O4f & $\prime \prime$ & 50 & 4 & $-0.2 \pm 4.5$ & - & - \\
IC 3568 & O5f & $\prime \prime$ & 50 & 3 & $-0.3 \pm 1.8$ & - & - \\
NGC 1535 & O3 & Irreg? & 70 & 3 & $-0.6 \pm 1.3$ & - & - \\
NGC 1360 & sdO & Const? & 80 & 7 & $-1.1 \pm 1.5$ & - & - \\
NGC 7009 & cont. & - & 82 & 6 & $-2.1 \pm 2.2$ & - & - \\
NGC 4361 & sdO & - & 82 & 3 & $+2.1 \pm 1.1$ & - & - \\
NGC 7293 & wD & " & 90 & 8 & $-1.5 \pm 1.1$ & - & - \\
NGC 6853 & wD & $\prime \prime$ & $>100$ & 2 & $-0.6 \pm 2.5$ & - & - \\
NGC 246 & C3/OVI & Const. & $>130$ & 4 & $-3.5 \pm 3.8$ & - & - \\
K1-16 & PG 1159 & Puls. & $>150$ & 10 & $+4.9 \pm 1.9$ & - & - \\
\hline
\end{tabular}

